\title{
RETRACTED ARTICLE: Convolution Operators Associated with the Generalized Airy Operator on the Complex Plane
}

\author{
Med Saber Ben Hammouda
}

Received: 9 May 2014 / Accepted: 23 February 2015 / Published online: 6 March 2015

(C) Springer Basel 2015

The article published online in Complex Analysis and Operator Theory, DOI 10.1007/ s11785-015-0451-1 has been retracted by the Publisher because it contains significant parts plagiarizing another publication: J.J. Betancor/M. Sifi/K. Trimeche, Hypercyclic and chaotic convolution operators associated with the Dunkl operator on C, Acta Math. Hung. 106 (2005), pp. 101-116, DOI 10.1007/s10474-005-0009-1.

The online version of this article contains the full text of the retracted article as electronic supplementary material.

Electronic supplementary material The online version of this article (doi:10.1007/s11785-015-0451-1) contains supplementary material, which is available to authorized users.

M. S. Ben Hammouda $(\varangle)$

Department of Mathematics, Faculty of Applied Sciences, Umm-Al-Qura University, AlAbdiya, P.O. Box 715, Makkah 21599, Saudi Arabia

e-mail: bhmsaber@gmail.com

M. S. Ben Hammouda

Département de Mathématiques, Faculté des Sciences de Tunis, Campus Universitaire Dél Menzah, 1060 Tunis, Tunisie 